\title{
1. Introduction: media and family interaction
}

\author{
Anja Riitta Lahikainen, Tiina Mälkiä and \\ Katja Repo
}

This book is a repository of new knowledge on how media and family activities intertwine with each other in daily family interaction and child socialization. In addition, it outlines the challenges and opportunities new media bring to family life and children's well-being. It addresses the question of how the digitalization of society and the changes in families' media environment influence family practices, such as family time, intergenerational interaction, and the participation of children and their time use.

The book discusses very timely and largely unexplored phenomena that are internationally identifiable. As a result of globalization, the digitalization of the living environments of children - such as the home has become an increasingly international and universal phenomenon. The digital revolution has also complicated family life, and family interaction worldwide is affected by the presence of various media devices. However, the implications and consequences of this transformation of family interaction are still largely unknown.

The research detailed in this volume shows how delicately media affect interaction between children and parents, and how profoundly they challenge everyday parenting. This kind of information is urgently needed, since the patterns of how media are encountered in the context of the family are still unstable and controversial. The book reveals the complexity and diversity of media-related family interaction by utilizing the unique data of video recordings of the family life of 26 Finnish families with a five- or a 12-year-old child. Video cameras were placed in their homes for one weekday to document the interaction around the kitchen table and in front of the child's main television and computer. Using the video data, we have been able to analyse what really happens in family homes instead of what parents think happened, or wished had happened.

The analyses in the book are based on the Finnish research project 
'Media, Family Interaction and Children's Well-being' funded by the Academy of Finland. This book will show that although the analysed data were collected in Finland, the situations and issues are universally recognizable. We are all aware of the interactional complexities of situations where parents want to catch the attention of a child who is playing a computer game, or when a child wants to get her or his parent's attention while the parent is focused on a smartphone. The case analyses demonstrate how profoundly new media changes interaction between children and parents. This change is applicable and identifiable universally.

In all, this book offers a novel and comprehensive picture of the complexity of the daily lives of contemporary families equipped with modern information and communication technologies. The issues are discussed from multiple perspectives. The first part of the book maps contemporary family life by providing methodological, theoretical and time-use reflections on media use and media-related family interaction. In addition, it discusses conversation analysis as a method for depicting the complexity of family interaction. This part utilizes time-use surveys as well as recent theoretical and methodological discussions.

In Chapter 2, Anja Riitta Lahikainen discusses theoretically how new media, family interaction and the socialization of children are intertwined and connected to each other. Lahikainen investigates what kind of challenges media present for the socialization of the child at home. Chapter 3 by Susan Danby focuses on family practices and media use. Danby's chapter is also theoretical, but instead of focusing on the aspects of socialization, her emphasis is on questions of social interaction and media use. In Chapter 4, Tiina Mälkiä introduces a new perspective, performativity, to family interaction studies. She demonstrates how the family members used the research cameras for their own amusement by creating expressive performances, and how the parents modified the image being recorded by producing moral performances. In the final chapter of the first part of the book, Chapter 5, Katja Repo and Satu Valkonen assess children's media use in relation to general time use. They focus on issues of access to media devices and the amount of time children spend with new media.

The second part of the book reaches into the private zone of family interaction, and provides the reader with detailed interactional analyses of everyday life with media devices. It contributes by offering detailed case studies of the various forms of media-related family interaction, new forms of family time, and conflict situations. This part utilizes the video recordings as data.

The second part begins with Chapter 6 by Aku Kallio on parental mediation in television viewing. Kallio explores parental mediation as an 
interactional phenomenon and investigates what sort of actions parental mediation can consist of and how mediation is realized in naturally occurring family interaction. In Chapter 7, Eero Suoninen focuses on the gender dimensions of interaction between parents and children in the context of watching television. His analysis includes two cases - one of father-son interaction and one of mother-daughter interaction - in which gender-related practices are prevalent. In Chapter 8, Suoninen continues by analysing the interaction that takes place in the context of playing a computer game. The analysis is based on a discussion between a parent and child in a situation where the child is strongly preoccupied with a computer game. In Chapter 9, Eerik Mantere and Sanna Raudaskoski introduce the concept of the 'sticky media device' and analyse a scenario in which a child is seeking her mother's attention when the mother is using a smartphone. In the last chapter of this section, Chapter 10, Sanna Tiilikainen and Ilkka Arminen focus on the ways families spend time together at home with and around media devices, combining individual and family time.

The concluding part of the book re-examines the themes presented in the empirical chapters. In Chapter 11, Sanna Raudaskoski, Eerik Mantere and Satu Valkonen discuss media use practices, interaction research, and theories of developmental psychology. They theorize the potential influences of parental smartphone use on child development. In the concluding chapter, Anja Riitta Lahikainen and Ilkka Arminen discuss the media-related risks, opportunities and new practices in late modern society. The final part of the book is completed by Peter Nikken's Commentary in Chapter 13 and in Chapter 14 by Jackie Marsh's Afterword. 
Anja Riitta Lahikainen, Tiina Mälkiä, and Katja Repo - 9781785366673 Downloaded from PubFactory at 04/26/2023 01:47:31PM via free access 